\title{
Localized Probing of Gas Molecule Adsorption Energies and Desorption Attempt Frequencies \\ Jared Cullen, ${ }^{\dagger}$ Alan Bahm,$\stackrel{\dagger}{\dagger}$ Charlene J. Lobo,${ }^{\dagger}$ Michael J. Ford, ${ }^{\dagger}$ and Milos Toth ${ }^{*, \dagger}$ \\ School of Physics and Advanced Materials, University of Technology, Sydney, 15 Broadway, Ultimo, New South Wales 2007, Australia, and FEI Company, 5350 NE Dawson Creek Dr, Hillsboro, OR 97124 USA \\ Received January 22, 2015; E-mail: Milos.Toth@uts.edu.au
}

\begin{abstract}
Gas-mediated electron beam induced etching (EBIE) and deposition (EBID) are often used to measure activation energies that are interpreted as the adsorption energies of surfaceadsorbed precursor molecules. However, the measured quantities often disagree with adsorption energies measured by conventional analysis techniques such as thermally programmed desorption, and have anomalous dependencies on parameters such as the electron beam current used to perform EBID. Here, we use the theory of EBIE and EBID rate kinetics to explain this behavior and identify conditions under which the activation energies and the associated pre-exponential factors correspond to gas molecule adsorption energies and desorption attempt frequencies, respectively. Under these conditions, EBIE and EBID can be used as robust, nano-scale techniques for the analysis of adsorbates.
\end{abstract}

\section{Introduction}

Gas-mediated electron beam induced etching (EBIE) and deposition (EBID) are direct-write nanofabrication techniques in which electrons dissociate surface-adsorbed precursor molecules, initiating chemical reactions on a solid substrate. ${ }^{1-3}$ The techniques are of interest for two main reasons. First, they enable beam-directed deposition, etching and surface functionalization with nanoscopic spatial resolution. ${ }^{4-13}$ Second, they can be used as analysis techniques for the characterization of adsorbates at surfaces, enabling the measurement of properties that include the gas molecule adsorption energy $\left(E_{a}\right)$, desorption attempt frequency $\left(k_{0}\right)$, diffusion coefficient, and electron dissociation cross-section. ${ }^{14-22} \mathrm{Un}$ like complimentary conventional techniques such as thermally programmed desorption, ${ }^{23,24}$ the use of EBIE and EBID as analysis techniques offers high spatial resolution, and the ability to perform measurements on adsorbates in the steady state at various pressures and temperatures.

Characterization of adsorbates by EBID and EBIE typically involves the measurement of deposition or etch rates as a function of one or more control parameters (e.g. electron beam flux, substrate temperature, precursor vapor pressure). The rates are then analyzed using methods based on models of EBIE/EBID rate kinetics to extract the adsorbate properties of interest. However, in practice, these analyses yield quantities that are often inconsistent with conventional measurement techniques, and have anomalous dependencies on the control parameters, raising questions about the validity of EBIE/EBID as adsorbate analysis techniques. ${ }^{14,25}$ A specific example discussed in the literature is the measurement of $E_{a}$ realized

${ }^{\dagger}$ School of Physics and Advanced Materials, University of Technology, Sydney, 15 Broadway, Ultimo, New South Wales 2007, Australia

${ }^{\ddagger}$ FEI Company, 5350 NE Dawson Creek Dr, Hillsboro, OR 97124 USA by Arrhenius analysis of electron beam induced deposition rates. A number of groups have reported that the value of $E_{a}$ measured by EBID is $\sim 2.5$ to 5 times lower than expected, ${ }^{15,19,22}$ and the surprising result that $E_{a}$ scales inversely with the electron beam current used to perform EBID. ${ }^{15,19}$ These observations were attributed to electron stimulated desorption. Here we show that Arrhenius analyses of EBID rates yield activation energies and pre-exponential factors that are expected to scale with current (and other parameters), and explain these dependencies using established models of EBID rate kinetics. We define conditions under which the activation energies and prefactors correspond to $E_{a}$ and $k_{0}$, respectively. Our results are also applicable to EBIE and illustrate that: (i) EBID and EBIE are indeed expected to enable meaningful, quantitative characterization of adsorbates, and (ii) the results of such analyses require careful interpretation in the context of mechanisms behind EBIE/EBID rate kinetics.

\section{Background Theory}

EBIE and EBID rates can be calculated by solving equations for the rate of change of concentration of precursor gas adsorbates at the substrate surface $\left(\partial N_{a} / \partial t\right):^{2,25}$

$$
\frac{\partial N_{a}}{\partial t}=\Lambda-\frac{N_{a}}{\tau}-\frac{\partial N_{\alpha}}{\partial t}+D_{a} \nabla^{2} N_{a},
$$

where $t$ is time and $a$ and $\alpha$ represent gas molecule adsorbates and their fragments generated by electron induced dissociation, respectively. $\frac{\partial N_{a}}{\partial t}$ is given by a sum of fluxes representing precursor adsorption $(\Lambda)$, desorption $\left(\frac{N_{a}}{\tau}\right)$, electron induced dissociation $\left(\frac{\partial N_{\alpha}}{\partial t}\right)$ and surface diffusion $\left(D_{a} \nabla^{2} N_{a}\right) . N$ is number density at the surface, $\tau$ is the adsorbate residence time at the surface, and $D_{a}$ is the diffusion coefficient. Adsorbate surface coverage $(\Theta)$ is typically assumed to be limited to 1 monolayer by the Langmuir isotherm:

$$
\begin{aligned}
& \Lambda=s F(1-\Theta), \\
& \Theta=A N_{a}
\end{aligned}
$$

where $s$ and $F$ are the gas molecule sticking coefficient and flux, respectively, and $A$ is the area of a single surface site. The rate of change of concentration of the fragments $\alpha$ is given by:

$$
\frac{\partial N_{\alpha}}{\partial t}=n \sigma f N_{a}
$$

where $f$ is electron flux, $\sigma$ is the effective cross-section ${ }^{1}$ for electron-induced dissociation of the adsorbates $a$, and $n$ is the number of fragments generated per adsorbate. The etch or deposition rate scales with $\frac{\partial N_{\alpha}}{\partial t}$. In the case of deposition, the vertical growth rate is given by:

$$
\frac{\partial h}{\partial t}=V_{\gamma} \sigma f N_{a},
$$

where $h$ is the deposit height, and $V_{\gamma}$ is the volume of a single molecule added to the substrate per adsorbate dissociated in the 
deposition reaction.

In cylindrical coordinates the following parameters are functions of the radial distance $(r)$ from the electron beam axis: $N_{a}, N_{\alpha}, \Lambda, \Theta$, $f$ and $h$. The gas molecule flux $(F)$ is typically assumed to be independent of $r$ and $t$ over the area irradiated by electrons (exceptions are discussed in ${ }^{26-28}$ ):

$$
F=\frac{P}{\sqrt{2 \pi m_{g} k_{B} T_{g}}},
$$

where $P$ is gas pressure, $m_{g}$ is the gas molecule mass, $k_{B}$ is Boltzmann's constant and $T_{g}$ is gas temperature.

\section{Simulation Details}

Modeling of gas-mediated electron beam induced processes is typically achieved by either continuum ${ }^{1-3}$ or Monte Carlo ${ }^{29-33}$ methods. The continuum approach entails solving differential equations such as Eqn. 1 to find $N_{a}$ as a function of time and space. It can be used to model processes of varying complexity, so long as the underlying physical and chemical mechanisms can be incorporated in Eqn. 1 (see, for example, the specific cases discussed in [14, 16, 34 39]). Monte Carlo models enable explicit simulation of individual electron-substrate and electron-adsorbate interactions. The primary advantage of the Monte Carlo approach is that it yields the interdependent time-evolution of the surface and the flux of electrons emitted from the surface. It is, however, limited by long computation times needed to simulate adsorbate transport over the spatial and temporal scales typically encountered in experiments.

Here we used a combination of these two techniques to implement the iterative, hybrid continuum-Monte Carlo model detailed in the Supporting Information. The hybrid model solves differential equations such as Eqn. 1 to find deposition (and/or etch) rates over time intervals between which the Monte Carlo method is used to calculate the resulting surface topology and the corresponding electron flux profile. It enables accurate calculation of the time evolution of structures grown by EBID/EBIE over the length and temporal scales needed to model real-world experiments.

The simulated system is EBID performed using cyclopentadienyl trimethyl platinum as the precursor gas. The growth of nano-pillars was simulated using a Gaussian electron beam with a diameter of $10 \mathrm{~nm}$, at substrate temperatures in the range of 250 to $450 \mathrm{~K}$ and an electron beam energy of $5 \mathrm{keV}$. All other model input parameters are provided in the Supporting Information. The pillar growth rates were measured in the steady state and analyzed to extract activation energies as detailed below.

\section{Results and Discussion}

\section{Arrhenius analysis of deposition rates}

The precursor molecule adsorption energy $\left(E_{a}\right)$ and attempt frequency $\left(k_{0}\right)$ can, in principle, be obtained by Arrhenius analysis of the growth rates of deposits made by EBID. However, this approach is valid only if the growth rates scale exponentially with reciprocal substrate temperature $(1 / T)$, and the scaling is caused solely by the temperature-dependence of the adsorption time $(\tau)$ :

$$
\tau=\frac{1}{k_{0}} \exp \left(\frac{E_{a}}{k_{B} T}\right) .
$$

Referring to Eqns. 1 and 5, Arrhenius analysis of growth rates therefore yields $E_{a}$ and $k_{0}$ only if:
(1) the adsorption flux $\Lambda$ is independent of $T$ over the range of $T$ used to perform the analysis (i.e. $\Theta \approx 0, d s / d T \approx 0$, and $d F / d T \approx 0$, so that $\Lambda \approx s F$ ), which we refer to as the 'athermal adsorption flux condition',

(2) the thermal desorption rate is much greater than the adsorbate dissociation rate (i.e. $\tau^{-1} \gg \sigma f$ ), which we refer to as the 'reaction-rate limited growth condition',

(3) net transport of adsorbates through diffusion is negligible (i.e. $\nabla^{2} N_{a} \approx 0$ ), which we refer to as the 'negligible diffusion condition', and

(4) the growth rate is measured in the steady state (i.e. $\left.\partial N_{a} / \partial t \approx 0\right)$, which we refer to as the 'steady state growth condition'.

Under these conditions, Eqns. 1 and 5 reduce to:

$$
\begin{gathered}
0=s F-\frac{N_{a}}{\tau}-\sigma f N_{a}, \\
\begin{aligned}
\frac{\partial h}{\partial t} & =\frac{V_{\gamma} \sigma f s F}{\tau^{-1}+\sigma f}, \\
& \approx V_{\gamma} \sigma f s F \tau,
\end{aligned}
\end{gathered}
$$

and Arrhenius analysis of $\partial h / \partial t$ yields $E_{a} / k_{B}$ and $V_{\gamma} \sigma f s F k_{0}^{-1}$ (i.e. both the adsorption energy $E_{a}$ and desorption attempt frequency $k_{0}$ can be deduced if the quantity $V_{\gamma} \sigma f s F$ is known). In the following sections, we analyze the effects of conditions 1-4 on the activation energies and prefactors obtained by Arrhenius analysis of EBID rates. We show that if any one of these conditions is not satisfied then Eqn. 10 is invalid and the values yielded by Arrhenius analysis diverge from $E_{a}$ and $k_{0}$.

\section{Athermal adsorption flux condition}

Gas molecules incident onto the substrate either adsorb to or reflect from the surface. The fraction that adsorb is given by the product $s(1-\Theta)$ in Eqn. 2, and the fraction that reflect is given by $s \Theta$. Hence, if the coverage $\Theta$ changes with $T$, then the rate at which gas molecules are reflected from occupied surface sites affects the temperature dependence of the adsorption flux $\Lambda$ which, in turn, affects $N_{a}(T), \partial h / \partial t(T)$ and the activation energy and prefactor yielded by Arrhenius analysis of EBID rates.

The surface coverage $\Theta$ increases with $P$ and $1 / T$ as shown in Fig. 1 for the platinum precursor considered in our simulations. The purple area indicates the range of $P$ and $T$ corresponding to the 'athermal adsorption flux condition', where $\Theta \approx 0$. In this part of the parameter space, the effect of $\Theta(T)$ on Arrhenius analysis of deposition rates is negligible. Outside this region, $\Theta \gg 0$ and the activation energy and prefactor obtained by Arrhenius analysis of $\partial h / \partial t$ both approach zero as $\Theta \rightarrow 1$. This is illustrated in Fig. 2 and 3 by plots of the activation energy and prefactor obtained as a function of $T$ (using a current density of $1.27 \times 10^{-7} \mathrm{nA} / \mathrm{nm}^{2}$ and a precursor pressure of $10 \mathrm{mPa}$ ). The shapes of the curves in Fig. 2 and 3 are governed by the functional form of the Langmuir isotherm used in Eqn. 2 (i.e. the dependence of $\Lambda$ on $\Theta$ ). Other isotherms will change the shapes of the curves, but will not make them independent of $\Theta$, except for the idealized special case of unlimited multilayer adsorption of non-interacting adsorbates in which $\Lambda=s F$.

The primary practical implication of the athermal adsorption flux condition is that Arrhenius analysis of EBID rates must be performed under conditions of negligible surface coverage because $\partial N_{a} / \partial T \rightarrow 0$ as $\Theta \rightarrow 1$ due to reflection of gas phase precursor 


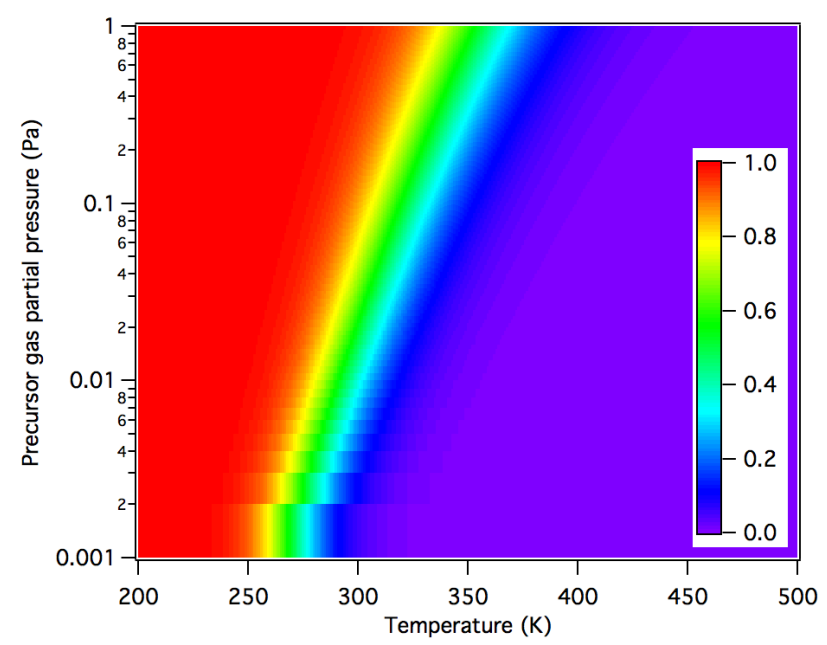

Figure 1. Precursor coverage $(\Theta)$ plotted as a function of pressure $(P)$ and substrate temperature $(T)$ in the limit of zero electron flux $(f \rightarrow 0)$. The purple region indicates the range of $P$ and $T$ over which the effect of $\Theta$ on Arrhenius analysis of EBID rates is negligible.

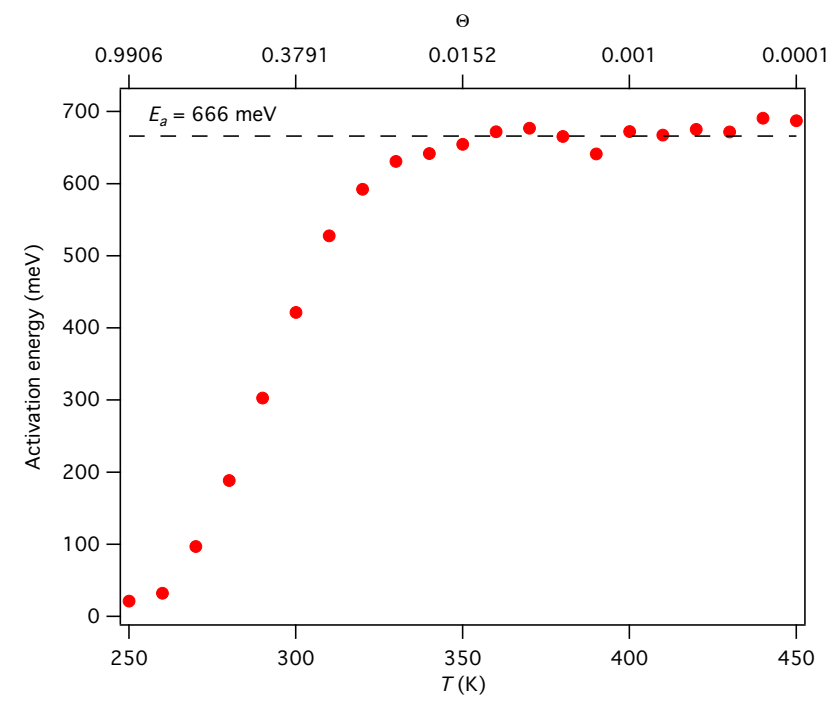

Figure 2. Activation energy obtained by Arrhenius analysis of the EBID rate $(\partial h / \partial t)$ simulated at a number of temperature windows between $250 \mathrm{~K}$ and $450 \mathrm{~K}$. The adsorption energy $\left(E_{a}\right)$ of $666 \mathrm{meV}$ is shown as a dashed line. The top axis shows the precursor coverage $(\Theta)$ corresponding to each temperature shown on the bottom axis. The activation energy diverges from $E_{a}$ as $\Theta \rightarrow$ 1. [Each datapoint was calculated from EBID rates simulated over a temperature window $\Delta T$ in the range of 20 to $50 \mathrm{~K}$.]

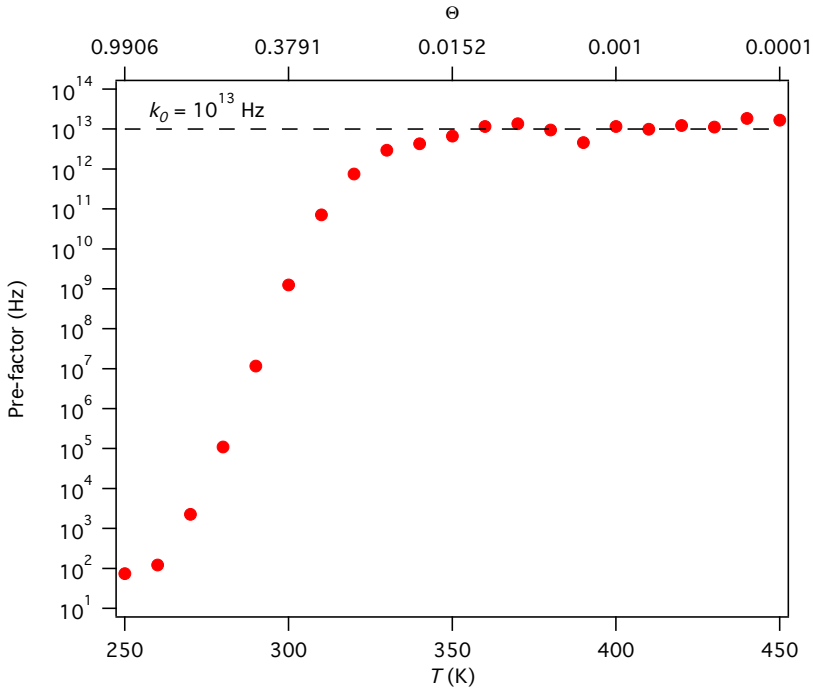

Figure 3. Prefactors corresponding to the activation energies shown in Fig. 2. The desorption attempt frequency $\left(k_{0}\right)$ of $10^{13} \mathrm{~Hz}$ is shown as a dashed line. The top axis shows the precursor coverage $(\Theta)$ corresponding to each temperature shown on the bottom axis. The prefactor diverges from $k_{0}$ as $\Theta \rightarrow 1$.

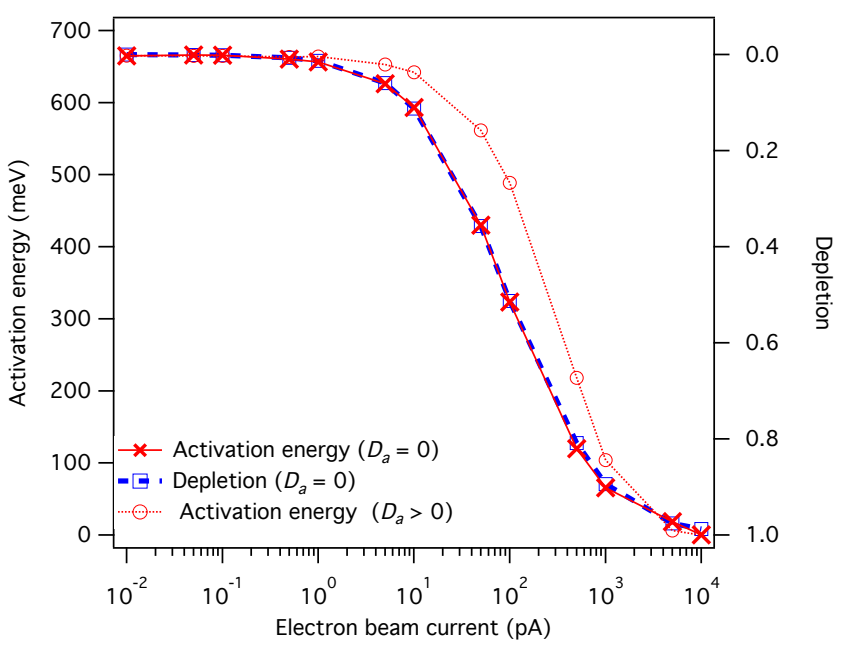

Figure 4. Dependence of activation energy on beam current simulated in the absence of diffusion $\left(D_{a}=0\right)$ and in the presence of diffusion $\left(D_{a}=D_{0} e^{-E_{d} /\left(k_{B} T\right)}\right.$, where $E_{d}$ is the diffusion energy). Also shown is a plot of adsorbate depletion $\left(\Theta_{r \rightarrow 0} / \Theta_{r \rightarrow \infty}\right)$ simulated at the beam axis $(r \rightarrow 0)$ in the absence of diffusion. The activation energy diverges from the adsorption energy $\left(E_{a}\right)$ of $666 \mathrm{meV}$ as the extent of depletion approaches 1 . The precursor pressure was $0.01 \mathrm{~Pa}$ and the temperature was varied from 400 to $450 \mathrm{~K}$. 
molecules from occupied surface sites. If $\Theta>0$ then both $E_{a}$ and $k_{0}$ will be underestimated by an amount that scales with $\Theta$ in a manner defined by the adsorption isotherm.

We note that since $\Lambda=s F(1-\Theta), s$ and $F$ must also not vary with $T$ in order for the athermal adsorption flux condition to be satisfied. The sticking coefficient can typically be assumed to be independent of $T$ (as has been done in our simulations). However, this assumption is not valid in some cases, such as that of activated sticking encountered in chemisorption. ${ }^{16}$ The gas molecule flux $F$ (given by Eqn. 6) is independent of substrate temperature $T$ if it is varied independently of the gas temperature $\left(T_{g}\right)$. This requirement is satisfied in most EBID experiments because standard EBID is a cold-wall deposition technique in which $T_{g}$ is dominated by the temperature of the capillary used to deliver the precursor gas.

\section{Reaction-rate limited growth condition}

The reaction-rate limited growth condition is violated (i.e. $\tau^{-1} \ngtr$ $\sigma f)$ if the rate at which adsorbates are consumed in the deposition reaction $(\sigma f)$ is significant relative to the thermal desorption rate. Consequently, Eqn. 10 is not a good approximation of Eqn. 9, and the activation energies and prefactors yielded by Arrhenius analyses of EBID rates diverge from $E_{a}$ and $k_{0}$, respectively. This is illustrated in Fig. 4 by a plot of the activation energy obtained as a function of beam current in the absence of diffusion (the simulation was performed with $D$ fixed at zero in order to delineate the effects of $\sigma f$ from the additional effects of diffusion which are discussed below). An increase in beam current causes an increase in the electron flux $(f)$ which, in turn, causes an increase in the adsorbate consumption rate in the deposition reaction $(\sigma f)$. If $\sigma f$ is significant relative to $\tau^{-1}$, then the denominator of Eqn. 9 is dominated by the temperature-independent $\sigma f$. Hence, the activation energies (and prefactors) yielded by Arrhenius analyses of EBID rates approach zero as $\sigma f \rightarrow \infty$. More specifically, in the absence of diffusion, the activation energy is directly proportional to the extent of depletion (given by $\Theta_{r \rightarrow 0} / \Theta_{r \rightarrow \infty}$ since $f=0$ at $r=\infty$ ). This is illustrated in Fig. 4 by a plot of $\Theta_{r \rightarrow 0} / \Theta_{r \rightarrow \infty}$, superimposed on the plot of activation energy versus beam current.

The primary practical consequence of the reaction-rate limited growth condition is that EBID must be performed in the so-called reaction rate limited growth regime ${ }^{2}$ defined by $\tau^{-1} \gg \sigma f$ (i.e. in the regime where electron irradiation does not cause significant depletion of precursor adsorbates).

\section{Negligible diffusion condition}

If $\nabla^{2} N_{a} \not 0$ then adsorbates are replenished not only through adsorption from the gas phase, but also via diffusion along the substrate surface. ${ }^{2}$ This is significant because any adsorbate replenishment mechanism that is distinct from adsorption alters the temperature-dependence of the coverage $\Theta$ and hence the activation energy (and prefactor) yielded by Arrhenius analysis of deposition rates.

In EBID, adsorbate replenishment through diffusion is negligible in the reaction rate limited growth regime ${ }^{2}$ because net adsorbate transport through diffusion requires an adsorbate concentration gradient. Hence, if the reaction-rate limited growth condition is satisfied (i.e. $\tau^{-1} \gg \sigma f$ ), then the negligible diffusion condition is automatically satisfied (i.e. $\nabla^{2} N_{a} \approx 0$ ). This is illustrated in Fig. 4 by the plot of activation energy versus beam current (simulated with diffusion activated in the model) which shows that the activation energy approaches $E_{a}$ as the extent of depletion approaches zero.

We note that in Fig. 4, the difference between the curves of activation energy versus beam current simulated with and without diffusion show that the net effect of diffusion is to decrease the dis- crepancy between the activation energy and $E_{a}$. This is expected since the net flow through diffusion acts to replenish adsorbates consumed in EBID and therefore alleviates depletion.

\section{Steady state growth condition}

In order to obtain $E_{a}$ and $k_{0}$, the Arrhenius analysis must be performed in the steady state (whereby $\frac{\partial N_{a}}{\partial t} \approx 0$ and $\partial h / \partial t$ is constant). If steady state has not been attained, the growth rate changes during the time interval over which $\partial h / \partial t$ is measured by an amount that is different at each substrate temperature, thereby altering the activation energies and prefactors obtained by the Arrhenius analysis of EBID growth rates. In practice, this is problematic only if the reaction rate limited growth condition is not satisfied, the growth time is too short, or some process such as electron beam damage modifies the deposit height while it is being grown by EBID. Experimentally, the condition of steady state growth can be verified by measuring deposit heights at each temperature versus time, and by showing that the rate of change of height is constant.

\section{General implications for determination of adsorbate properties by EBID and EBIE}

The overarching qualitative implication of the first three conditions (athermal adsorption flux, reaction-rate limited growth and negligible diffusion) is that the activation energy measured by EBID is lower than $E_{a}$ if any one of the conditions is violated. The discrepancy occurs if the increase in adsorbate concentration with reciprocal temperature is smaller than expected from the thermal desorption rate $\tau^{-1}$. Violations of one or more of these conditions likely account for the fact that activation energies reported in the EBID literature are often lower than $E_{a}$, but never greater than $E_{a} \cdot{ }^{15,19,22}$

Our general conclusions are also applicable to EBIE, which is analogous to EBID, except that Eqn. 5 represents the etch pit depth rather than the deposit height. However, the above analysis is strictly applicable only to systems in which adsorption is described by a single potential well. In cases such as activated chemisorption, which are described by multiple potential wells and one or more adsorption barriers, ${ }^{16,39,40}$ the above analysis must be re-done using appropriate rate equations in order to determine the correct meaning and scaling of activation energies and prefactors obtained in different temperature regimes. Care must also be taken to ensure that mechanisms that are not accounted for by Eqn. 1 do not alter the temperature dependence of $N_{a}$. For example, in EBIE, a number of such special cases have been identified. ${ }^{14,35-38}$ In these instances, the meaning of the measured activation energies and prefactors must be evaluated on a case-by-case basis, using the appropriate rate equations.

It should be noted that, in the present work, Arrhenius analysis was applied to vertical deposition rates at the beam axis (i.e. $\left.|\partial h / \partial t|_{r \rightarrow 0}\right)$. Our conclusions are, however, also applicable to Arrhenius analysis of all other measures of growth rate such as the deposit volume ${ }^{19}$ and mass. ${ }^{15}$ However, in these cases, the steady state growth condition is more stringent because steady state is attained only once the deposit shape stops changing due to the timeevolution of the electron interaction volume inside a growing deposit (see, for example, the discussions of the time-evolution of deposit shapes and base diameters in $[29,31,41])$.

Finally, we note that, qualitatively, the decrease in activation energy with increasing beam current seen in Fig. 4 has been observed experimentally. ${ }^{15,19}$ It was, however, attributed to electron stimulated desorption (ESD) rather than the phenomenon of adsorbate 
depletion which is inherent to EBID. ESD causes the adsorbate concentration $\left(N_{a}\right)$ to decrease at a rate $\sigma_{E} f$, where $\sigma_{E}$ is the crosssection for ESD. ${ }^{24,42-44}$ It can be incorporated into Eqn. 1 in the form of the ESD flux $\sigma_{E} f N_{a}$ :

$$
\frac{\partial N_{a}}{\partial t}=\Lambda-\left(\frac{N_{a}}{\tau}+\sigma_{E} f N_{a}\right)-\frac{\partial N_{\alpha}}{\partial t}+D_{a} \nabla^{2} N_{a},
$$

and therefore alters the denominator of Eqn. 9:

$$
\frac{\partial h}{\partial t}=\frac{V_{\gamma} \sigma f s F}{\tau^{-1}+\sigma_{E} f+\sigma f} .
$$

Consequently, the ESD rate $\sigma_{E} f$ affects Arrhenius analysis by contributing to adsorbate depletion in the same manner as the dissociation rate $\sigma f$. However, the extent of ESD is typically negligible since, for most adsorbates, $\sigma_{E}$ lie in the range ${ }^{24} 10^{-7} \AA^{2}$ to $10^{-2} \AA^{2}$ (i.e., in general, $\sigma_{E} \ll \sigma$ ). We therefore conclude that the decrease in activation energy with beam current observed by Li et al. ${ }^{19}$ and van Dorp et al. ${ }^{15}$ is expected from the net effect of $\sigma f+\sigma_{E} f$ even if $\sigma_{E}$ is negligible. It is caused by an increase in the extent of depletion of precursor adsorbates with increasing electron flux (i.e. violation of the reaction rate limited growth condition), irrespective of whether or not ESD plays a significant role in EBID.

\section{Conclusion}

We have developed a hybrid continuum-Monte Carlo model of electron beam induced etching and deposition that enables accurate calculation of the growth of nano- and micro-structures over the length and time scales used in experiments. The model was used to simulate the dependencies of EBID rates on experimentally controlled growth parameters, and to interpret the physical meaning of activation energies and pre-exponential factors obtained by Arrhenius analysis of EBID rates. The activation energies were shown to correspond to precursor molecule adsorption energies, and the prefactors to desorption attempt frequencies, provided that EBID is performed under specific conditions. We have shown how deviations from these conditions affect the Arrhenius analysis and explained the observed trends as being caused by changes in adsorbate concentration with key EBID experimental parameters.

\section{Supporting Information Avail- able}

Details on the Hybrid Continuum-Monte Carlo model and precursor specific input parameters used in all simulations are available in the supporting information. This material can be found at http://pubs.acs.org.

\section{Supporting Information I: Hy- brid continuum-Monte Carlo Model}

Here, we describe the hybrid continuum-Monte Carlo model used to perform EBID simulations. The model calculates precursor concentrations and deposition (or etch) rates using a continuum rate equation approach, and the time-evolution of the electron flux profile by Monte Carlo simulations of electron-solid interactions. Iterative application of these two methods enables efficient simulation of changes in surface geometry with time, as well as the effects of surface evolution on the electron flux and adsorbate concentration profiles. EBID rate equations were solved numerically using the Crank-Nicolson method in cylindrical coordinates. Monte Carlo simulations were performed in Cartesian coordinates.

\section{Surface evolution and diffusion}

The initial flat substrate surface was discretized with $0.1 \mathrm{~nm}$ resolution and updated periodically using $\partial h / \partial t(r, t)$, obtained using Eqn. 5. However, in order to evolve the surface correctly, the deposition (or etch) rates must be applied along the surface normal, rather than the vertical direction. In the simplest case, the surface normal can be calculated from the vector joining the nearest neighbors of the point that is to be moved. However, to mitigate the effects of noise generated by Monte Carlo calculation of the electron flux profile, we calculate the surface normal over an 11 point window. This prevents sharp spikes and other anomalous effects from generating artifacts in the surface geometry.

Once the surface geometry is updated at each time step, the surface is re-discretized such that each annulus making up the surface remains constant. This is required so that the annuli in the diffusion term of Eqn. 1 are constant, and the form of the Laplacian operator is maintained regardless of surface topography.

\section{Electron beam projection}

As the surface evolves and the sidewalls of the deposited or etched structure grow, the surface area intersected by the primary electron beam changes, requiring recalculation of the electron flux profile (achieved by projecting the electron beam onto the growing surface at each time step of the simulation). It is important to note that the backscattered and secondary electron flux profiles are not modified in this manner as they are calculated explicitly by the Monte Carlo algorithm described below.

\section{Monte Carlo model of electron-solid in- teractions}

Monte Carlo models are able to simulate all of the individual electron interactions with the substrate and with adsorbates, and the resultant time-evolution of the surface. They are, however, limited by the long computation times needed to simulate the transport of individual precursor molecules over the spatial and temporal scales encountered in experiments. The length scale that must be simulated can be approximated by the sum of the electron beam diameter, the diameter of the electron interaction volume in the substrate and the diffusion length of adsorbates.

Here we used the Monte Carlo approach ${ }^{45,46}$ to model electronsolid interactions and to obtain the backscattered and secondary electron contributions to the total electron flux profile $f(r, t)$. However, we did not use it to simulate electron-adsorbate interactions, thereby eliminating the large computational overhead associated with the simulation of adsorbate transport within the Monte Carlo method. Instead, Eqn. 1 is used to simulate electron-adsorbate interactions and adsorbate transport over short time intervals, between which $f(r, t)$ is updated periodically by the Monte Carlo method. The magnitude of the time interval provides control over the tradeoff between computation time and accuracy. For the simulations presented here, the initial time step was $1 \mathrm{~ns}$, and it was increased by $10 \%$ at every time step (as the system approached a steady state) up to a maximum of $10 \mathrm{~ms}$. 
Our Monte Carlo model uses Rutherford scattering crosssections and the continuous slowing down approximation. ${ }^{45}$ The trajectories of forward and backscattered electrons are calculated using a line-line intersection method, ${ }^{47}$ which involves calculating the intersection point of the current electron scattering event and each point on the surface. When only one intersection point lies on the surface the electron is said to have backscattered through this point and left the solid. When two intersection points lie on the surface the electron is said to have forward scattered out of the surface at the first intersection point (e.g. a pillar sidewall), and into the surface at the second intersection point (e.g. a horizontal substrate region adjacent to the pillar). Secondary electrons are not simulated explicitly but instead calculated using a modified version of the parametric model designed by Joy. ${ }^{45}$ The parametric model approximates the secondary electron yield at a single point on a flat surface and enables accurate modeling of secondary electron generation without requiring details of their generation or scattering cascades. In our implementation, the parametric model was modified to enable SE generation from curved surfaces. The first modification was to evenly distribute the generated secondary electrons along the entire primary electron path between elastic scattering events. Second, we assumed isotropic SE generation, and calculated the fraction that intersects each point making up the discretized surface.

\section{Model verification}

\section{Continuum code verification}

To verify our implementation of the continuum EBID component of the hybrid model, a test case was developed to compare it to a model published previously by Lobo et al. ${ }^{48}$ The latter simulates electron beam induced etching and deposition arising from a mixture of etch and deposition precursor gases. It reduces to our model of EBID when the etch gas pressure is set to zero.

To compare the models, the growth rates of a series of deposits were simulated as a function of electron beam current. Fig. 5 shows that the vertical growth rate calculated at $r \sim 1.5 \AA$ decreases with increasing current. The decrease in growth rate is caused by adsorbate depletion near the beam axis. The excellent agreement between the two models seen in the figure confirms consistent implementation of all terms in Eqn. 1.

\section{Monte Carlo code verification}

To verify our implementation of the Monte Carlo component of the hybrid model, it was tested against the well-established CASINO code $^{46}$ and experimental data. The tests were conducted using a silver substrate and electron beam energies in the range of 0.03 and $30.0 \mathrm{keV}$.

The first test compares the dependence of backscattered electron coefficient on electron beam energy calculated by our model to that calculated by CASINO. The results, shown in Figure 6, are in excellent agreement.

The second test compares the secondary electron yield calculated as a function of electron beam energy to experimental data. ${ }^{49}$ The simulation results shown in Fig. 7 are in good agreement with experiment at beam energies greater than $\sim 1 \mathrm{keV}$. The divergence from the experimental data at the lower beam energies is expected because of limitations inherent to the Rutherford cross sections and the slowing down approximation implemented in our model.

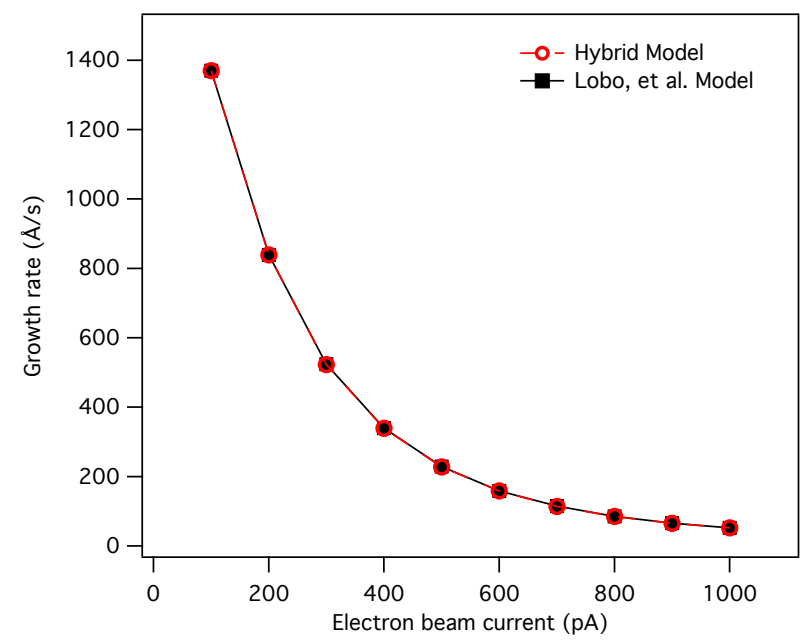

Figure 5. Comparison between the continuum component of the hybrid model used here and the continuum model published previously by Lobo et al. ${ }^{48}$ The EBID rates were calculated at a distance of $\sim 1.5 \AA$ from of the electron beam axis as a function of electron beam current. Both models show the same decrease in growth rate with increasing current, caused by adsorbate depletion near the beam axis.

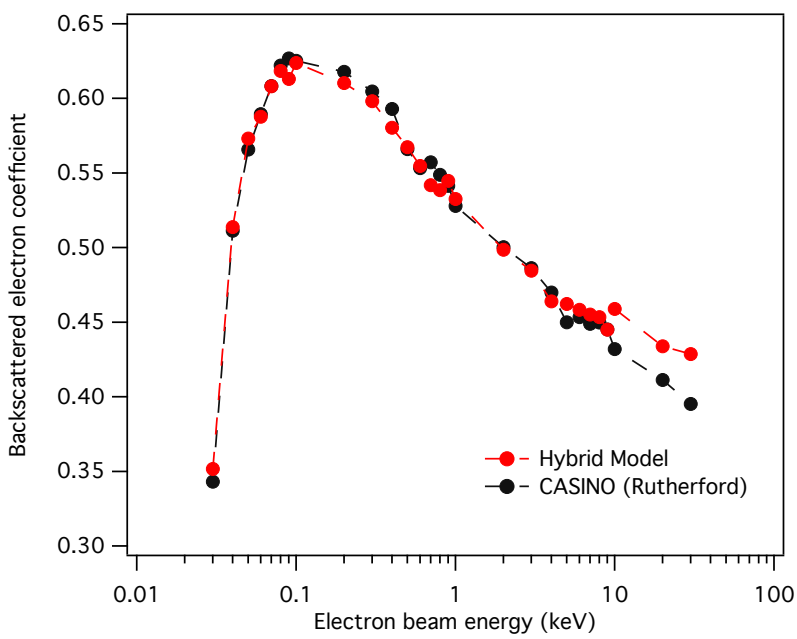

Figure 6. Comparison between the Monte Carlo component of the hybrid model used here and the Monte Carlo model CASINO. ${ }^{46}$ The dependence of the backscattered electron coefficient on electron beam energy calculated the hybrid model is in excellent agreement with that calculated by CASINO.

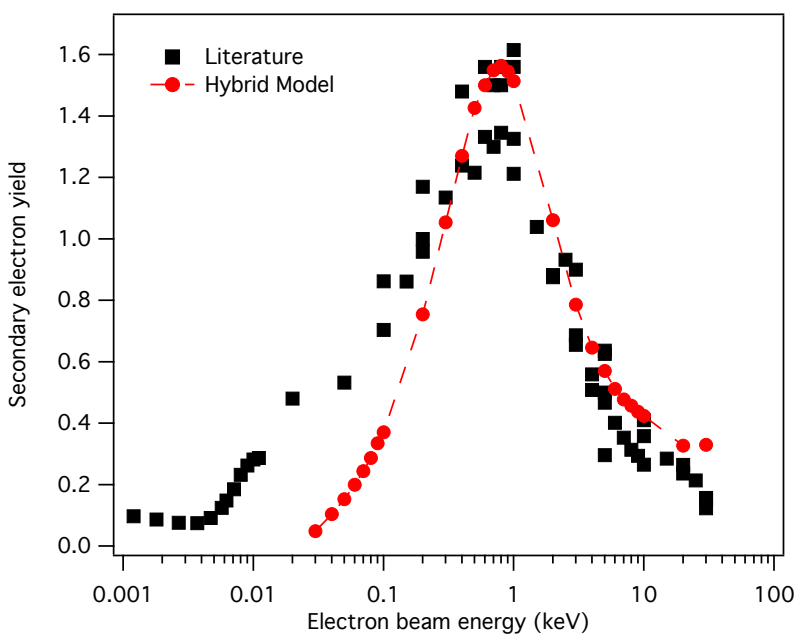

Figure 7. Comparison between the secondary electron yield on a silver surface as a function of electron beam energy taken from literature data and the hybrid continuum-Monte Carlo model. 


\section{Hybrid model verification}

To test the time-evolution of surfaces generated by the hybrid continuum-Monte Carlo model, we compared the volumes of deposits calculated by integration of the simulated surfaces ('actual volumes') to the corresponding total volumes of molecules deposited by electrons ('expected volumes'). The expected volumes are given by the product of $V_{\gamma}$ and the total number of molecules dissociated by electrons (which is generated automatically by the continuum component of the model since it solves Eqn. 1 to find $\left.f N_{a}(r, t)\right)$.

As shown in Fig. 8, the time-evolutions of the actual and expected volumes are in excellent agreement. The residual error, also shown on the plot, increases with simulation time. The error is controlled by the number of electrons simulated in each iteration of the Monte Carlo model, and the magnitude of the time step used to propagate the hybrid model in time.

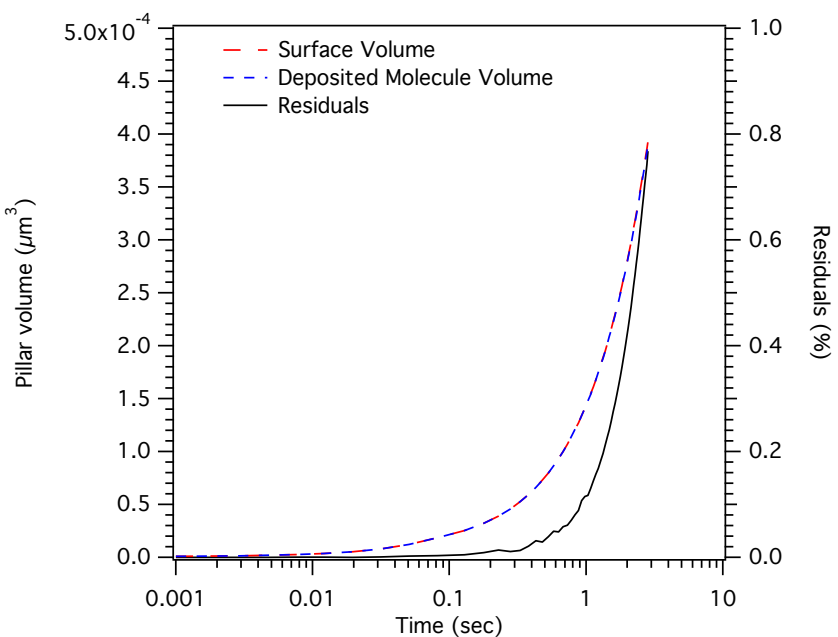

Figure 8. A comparison of the time-evolution of the actual volume of a deposit simulated by the hybrid model, and the volume expected from the total number of molecules dissociated by electrons. The two volumes are in excellent agreement, with only minor differences of $<1 \%$ observed in the residuals.

\section{Supporting Information II: Sim- ulation input parameters}

The following is a list of simulation input parameters relating to the electron beam and precursor molecules (the substrate and deposited material were assumed to have the same density and composition). We note that while a number of these values are approximations, the trends discussed in the paper are independent of the exact, quantitative values of the input parameters.

\section{Electron Beam}

- Diameter, $10.0 \mathrm{~nm}$

- Energy, $5.0 \mathrm{keV}$

- Current, $10 \mathrm{pA}$ to $10 \mathrm{nA}$

\section{Precursor Molecule}

- Atomic number, $78.0(\mathrm{Pt})$

- Atomic weight, $195.084 \mathrm{amu}\left(\mathrm{PtC}_{5}\right)$
- Density, $21.45 \mathrm{~g} / \mathrm{cm}^{3}\left(\mathrm{PtC}_{5}\right)$

- Energy required to produce a $\mathrm{SE}, 0.03 \mathrm{keV}(\mathrm{Pt})^{50}$

- SE escape depth, ${ }^{50} 5.0 \AA$

- Pressure, $10 \mathrm{mPa}$

- Surface area, ${ }^{26} 35.7 \AA^{2}$

- Sticking coefficient, ${ }^{26} 1.0$

- Net electron dissociation cross section, $1.0 \AA^{2}$

- Desorption energy, ${ }^{51} 666 \mathrm{meV}$

- Desorption prefactor, ${ }^{52} 10^{13} \mathrm{~Hz}$

- Diffusion energy, ${ }^{51} 0.114 \mathrm{eV}$

- Diffusion prefactor, ${ }^{26} 4.16 \times 10^{9} \mathrm{~A}^{2} / \mathrm{s}$

\section{References}

(1) Toth, M. Advances in Gas-mediated Electron Beam-induced Etching and Related Material Processing Techniques. Applied Physics A 2014, 117 , $1623-1629$

(2) Utke, I.; Moshkalev, S.; Russell, P. Nanofabrication Using Focused Ion and Electron Beams; Principles and Applications; Oxford University Press, USA, 2012.

(3) Huth, M.; Porrati, F.; Schwalb, C.; Winhold, M.; Sachser, R.; Dukic, M.; Adams, J.; Fantner, G. Focused Electron Beam Induced Deposition: A Perspective. Beilstein Journal of Nanotechnology 2012, 3, 597-619.

(4) De Teresa, J. M.; Córdoba, R. Arrays of Densely Packed Isolated Nanowires by Focused Beam Induced Deposition plus Ar+ Milling. ACS Nano 2014, 8, 3788-3795.

(5) Kim, S.; Kulkarni, D. D.; Davis, R.; Kim, S. S.; Naik, R. R.; Voevodin, A. A.; Russell, M.; Jang, S. S. Tsukruk, V. V.; Fedorov, A. G. Controlling the Physicochemical State of Carbon on Graphene Using Focused Electron-Beam-Induced Deposition. ACS Nano 2014, 8, 6805-6813

(6) Shanley, T. W.; Martin, A. A.; Aharonovich, I.; Toth, M. Localized Chemical Switching of the Charge State of Nitrogen-Vacancy Luminescence Centers in Diamond. Appl. Phys. Lett. 2014, 105, 063103.

(7) Winhold, M.; Weirich, P. M.; Schwalb, C. H.; Huth, M. Superconductivity and Metallic Behavior in PbxCyO Structures Prepared by Focused Electron Beam Induced Deposition. Applied Physics Letters 2014, 105.

(8) Martin, A. A.; Toth, M.; Aharonovich, I. Subtractive 3D Printing of Optically Active Diamond Structures. Sci. Rep. 2014, 4, 5022.

(9) Fernández-Pacheco, A.; Serrano-Ramón, L.; Michalik, J. M.; Ibarra, M. R.; De Teresa, J. M.; O'Brien, L.; Petit, D.; Lee, J.; Cowburn, R. P. Three Dimensional Magnetic Nanowires Grown by Focused Electron-beam Induced Deposition. Sci. Rep. 2013, 3.

(10) Jenke, M. G.; Lerose, D.; Niederberger, C.; Michler, J.; Christiansen, S. Utke, I. Toward Local Growth of Individual Nanowires on ThreeDimensional Microstructures by Using a Minimally Invasive Catalyst Templating Method. Nano Lett. 2011, 11, 4213-4217.

(11) Walz, M.; Schirmer, M.; Vollnhals, F.; Lukasczyk, T.; Steinruck, H. P. Marbach, H. Electrons as "Invisible Ink": Fabrication of Nanostructures by Local Electron Beam Induced Activation of SiOx. Angew Chem Int Edit 2010, 49, 4669-4673.

(12) Toth, M.; Lobo, C. J.; Knowles, W. R.; Phillips, M. R.; Postek, M. T.; Vladar, A. E. Nanostructure Fabrication by Ultra-High-Resolution Environmental Scanning Electron Microscopy. Nano Lett. 2007, 7, 525-530.

(13) van Dorp, W. F.; van Someren, B.; Hagen, C. W.; Kruit, P.; Crozier, P. A Approaching the Resolution Limit of Nanometer-Scale Electron BeamInduced Deposition. Nano Letters 2005, 5, 1303-1307.

(14) Martin, A. A.; Toth, M. Cryogenic Electron Beam Induced Chemical Etching. ACS Appl. Mater. Interfaces 2014, 6, 18457-18460.

(15) van Dorp, W. F.; Hansen, T. W.; Wagner, J. B.; De Hosson, J. T. M. The Role of Electron-Stimulated Desorption in Focused Electron Beam Induced Deposition. Beilstein Journal of Nanotechnology 2013, 4, 474480.

(16) Bishop, J.; Lobo, C. J.; Martin, A.; Ford, M.; Phillips, M. R.; Toth, M. The Role of Activated Chemisorption in Electron Beam Induced Deposition. Phys. Rev. Lett. 2012, 109, 146103.

(17) Fowlkes, J. D.; Rack, P. D. Fundamental Electron-Precursor-Solid Interactions Derived from Time-Dependent Electron-Beam-Induced Deposition Simulations and Experiments. ACS Nano 2010, 4, 1619-1629.

(18) Utke, I.; Friedli, V.; Purrucker, M.; Michler, J. Resolution in focused electron- and ion-beam induced processing. Journal of Vacuum Science and Technology B: Microelectronics and Nanometer Structures 2007, 25, 2219-2223.

(19) Li, W.; Joy, D. C. Study of Temperature Influence on Electron Beam Induced Deposition. Journal of Vacuum Science E Technology A: Vacuum, Surfaces, and Films 2006, 24, 431. 
(20) Randolph, S. J.; Fowlkes, J. D.; Rack, P. D. Effects of Heat Generation During Electron-Beam-Induced Deposition of Nanostructures. Journal of Applied Physics 2005, 97, 124312.

(21) Scheuer, V.; Koops, H.; Tschudi, T. Electron Beam Decomposition of Carbonyls on Silicon. Microelectronic Engineering 1986, 5, 423-430.

(22) Christy, R. W. Formation of Thin Polymer Films by Electron Bombardment. Journal of Applied Physics 1960, 31, 1680.

(23) Zhdanov, V. P. Arrhenius Parameters For Rate-Processes On SolidSurfaces. Surf. Sci. Rep. 1991, 12, 183-242.

(24) Madey, T. E.; Yates Jr, J. T. Desorption Methods As Probes of Kinetics and Bonding at Surfaces. Surface Science 1977, 63, 203-231.

(25) Utke, I.; Hoffmann, P.; Melngailis, J. Gas-Assisted Focused Electron Beam and Ion Beam Processing and Fabrication. Journal of Vacuum Science $\mathcal{E}$ Technology B: Microelectronics and Nanometer Structures 2008, 26, 1197.

(26) Winkler, R.; Fowlkes, J.; Szkudlarek, A.; Utke, I.; Rack, P. D.; Plank, H. The Nanoscale Implications of a Molecular Gas Beam during Electron Beam Induced Deposition. ACS Applied Materials $\mathcal{E}$ Interfaces 2014, 6, 2987-2995.

(27) Friedli, V.; Utke, I. Optimized Molecule Supply from Nozzle-Based Gas Injection Systems for Focused Electron- and Ion-beam Induced Deposition and Etching: Simulation and Experiment. J. Phys. D 2009, 42, 125305.

(28) Bret, T.; Utke, I.; Hoffmann, P. Influence of the Beam Scan Direction During Focused Electron Beam Induced Deposition of 3D Nanostructures. $\mathrm{Mi}$ croelectronic Engineering 2005, 78-79, 307-313.

(29) Smith, D. A.; Fowlkes, J. D.; Rack, P. D. Understanding the Kinetics and Nanoscale Morphology of Electron-Beam-Induced Deposition via a Three-Dimensional Monte Carlo Simulation: The Effects of the Precursor Molecule and the Deposited Material. Small 2008, 4, 1382-1389.

(30) Smith, D. A.; Fowlkes, J. D.; Rack, P. D. Simulating the Effects of Surface Diffusion on Electron Beam Induced Deposition via a Three-Dimensional Monte Carlo Simulation. Nanotechnology 2008, 19, 415704.

(31) Smith, D. A.; Fowlkes, J. D.; Rack, P. D. A Nanoscale Three-Dimensiona Monte Carlo Simulation of Electron-Beam-Induced Deposition with Gas Dynamics. Nanotechnology 2007, 18, 265308

(32) Liu, Z.-Q.; Mitsuishi, K.; Furuya, K. A Dynamic Monte Carlo Study of the in situ Growth of a Substance Deposited Using Electron-Beam-Induced Deposition. Nanotechnology 2006, 17, 3832-3837.

(33) Silvis-Cividjian, N.; Hagen, C. W.; Kruit, P. Spatial Resolution Limits in Electron-Beam-Induced Deposition. Journal of Applied Physics 2005, 98, 084905.

(34) Bernau, L.; Gabureac, M.; Erni, R.; Utke, I. Tunable Nanosynthesis of Composite Materials by Electron-Impact Reaction. Angew Chem Int Edit 2010, 49, 8880-8884.

(35) Lassiter, M. G.; Rack, P. D. Nanoscale Electron Beam Induced Etching A Continuum Model That Correlates the Etch Profile to the Experimental Parameters. Nanotechnology 2008, 19, 455306.

(36) Martin, A. A.; Phillips, M. R.; Toth, M. Dynamic Surface Site Activation: A Rate Limiting Process in Electron Beam Induced Etching. ACS Appl. Mater. Interfaces 2013, 5, 8002-8007.

(37) Randolph, S.; Toth, M.; Cullen, J.; Chandler, C.; Lobo, C. Kinetics of Gas Mediated Electron Beam Induced Etching. Applied Physics Letters 2011 99, 213103

(38) Toth, M. Lobo, C. J ; Hartigan, G.; Ralph Knowles, W. Electron Flux Controlled Switching Between Electron Beam Induced Etching and Deposition. Journal of Applied Physics 2007, 101, 054309.

(39) Randolph, S. J.; Botman, A.; Toth, M. Deposition of Highly Porous Nanocrystalline Platinum on Functionalized Substrates Through FluorineInduced Decomposition of $\mathrm{Pt}\left(\mathrm{PF}_{3}\right)_{4}$ Adsorbates. Particle $\mathcal{E}$ Particle Systems Characterization 2013, 672-677.

(40) Bishop, J.; Toth, M.; Phillips, M.; Lobo, C. Effects of Oxygen on Electron Beam Induced Deposition of $\mathrm{SiO}_{2}$ Using Physisorbed and Chemisorbed Tetraethoxysilane. Appl. Phys. Lett. 2012, 101, 211605.

(41) Choi, Y. R Y; Rack, P. D. P.; Randolph, S. J. S. Smith, D. A. D.; Joy, D. C. D. Pressure Effect of Growing with Electron Beam-Induced Deposition with Tungsten Hexafluoride and Tetraethylorthosilicate Precursor. Scanning 2006, 28, 311-318.

(42) Madey, T.; Yates, J. T. Electron-Stimulated Desorption As a Tool For Studies of Chemisorption: A Review. Journal of Vacuum Science $\mathcal{E}$ Technology 1971, 8, 525-555.

(43) Drinkwine, M. J.; Lichtman, D. Electron Stimulated Desorption: A Critical Review. Progress in Surface Science 1977, 8, 123-142.

(44) Ramsier, R. D.; Yates, J. T. Electron-Stimulated Desorption - Principles and Applications. Surface Science Reports 1991, 12, 243-378.

(45) Joy, D. C. Monte Carlo Modeling for Electron Microscopy and Microanalysis; Oxford University Press, USA, 1995.

(46) Hovington, P.; Drouin, D.; Gauvin, R. CASINO: A New Monte Carlo Code in C Language for Electron Beam Interaction-Part I: Description of the Program. Scanning 1997, 19, 1-14.

(47) De Berg, M.; Van Kreveld, M.; Overmars, M.; Schwarzkopf, O. C. Computational geometry; Springer, 2000.

(48) Lobo, C. J.; Toth, M.; Wagner, R.; Thiel, B. L.; Lysaght, M. High Resolution Radially Symmetric Nanostructures from Simultaneous Electron Beam Induced Etching and Deposition. Nanotechnology 2007, 19, 025303.

(49) Joy, D. C. A Database on Electron-Solid Interactions. Scanning 1995, 17 270-275.

(50) Lin, Y.; Joy, D. C. A New Examination of Secondary Electron Yield Data Surface and Interface Analysis 2005, 37, 895-900.

(51) Shen, J.; Muthukumar, K.; Jeschke, H. O.; Valenti, R. Physisorption of an Organometallic Platinum Complex on Silica: An Ab Initiostudy. New Journal of Physics 2012, 14, 073040.

(52) Fichthorn, K.; Miron, R. Thermal Desorption of Large Molecules from Solid Surfaces. Physical Review Letters 2002, 89, 196103. 
Graphical TOC Entry

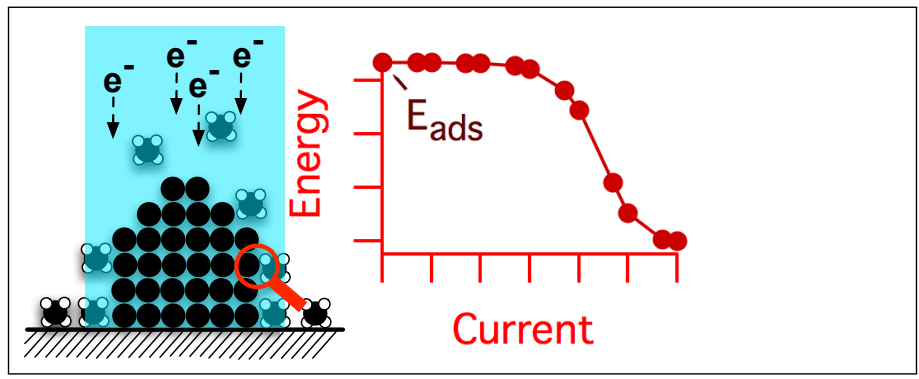

